\title{
Heart Failure and Angiotensin-Converting Enzyme Inhibitors
}

\author{
Is There a Need for Specialty Care?
}

C ongestive heart failure (CHF) causes significant morbidity and mortality in the United States. As of 1995, 4.7 million people in this country had been diagnosed with CHF. The 6-year mortality rate reaches $80 \%$ in men and $65 \%$ in women. ${ }^{1}$ Over the last 10 years, advances in our understanding of heart failure have led to new therapeutic developments. One such therapy is the angiotensin-converting enzyme (ACE) inhibitor.

In the late 1980s to early 1990s several pivotal, randomized controlled trials showed the benefit of such therapy in CHF. One of the early trials was the SOLVD trial, which showed that enalapril, added to conventional therapy, significantly reduced mortality and hospitalizations in patients with decreased left ventricular function. ${ }^{2}$ At the same time, the SOLVD investigators evaluated the effect of enalapril on mortality and morbidity in asymptomatic patients with reduced left ventricular function, and demonstrated an $8 \%$ reduction in mortality and a $37 \%$ reduction in the development of heart failure. ${ }^{3}$ Other studies have confirmed the substantial benefit of using ACE inhibitors in other patient groups, including those with $\mathrm{CHF}$ after myocardial infarction. ${ }^{4}$

Despite the overwhelming evidence that ACE inhibitors reduce morbidity and mortality in patients with left ventricular dysfunction and $\mathrm{CHF}$, studies indicate that this family of drugs is being underutilized. ${ }^{5,6}$ Furthermore, even when used, it is at lower doses than those shown to be effective in randomized trials. However, little is known about why the drug is underused and possibly underdosed and whether physician subspecialty affects the use of ACE inhibitors.

In this issue, Chin et al. provide important information on the underutilization of ACE inhibitors and the possible effects of physicians' speciality on the use of these agents. In their Brief Report, the authors performed a retrospective analysis of 214 outpatients with decreased systolic function treated at an urban medical center. ${ }^{7}$ They compared patients under the care of cardiologists versus generalist physicians versus a combination of both. Regardless of specialty, approximately $75 \%$ of physicians' patients were taking an ACE inhibitor. This percentage is higher than previous reports, which indicated that ACE inhibitors are used in only $30-40 \%$ of patients with heart failure. ${ }^{8}$ However, only $60 \%$ were taking doses that were proven to be efficacious in randomized trials. ${ }^{7}$
The major limitation of this retrospective study is that the indications for therapy were not measured. The generalist physicians' patients were more likely to have hypertension than the cardiologists' patients. This difference might indicate that the ACE inhibitors were being used for different indications: hypertension for generalists' patients and CHF for cardiologists' patients. It is difficult, therefore, to conclude that generalist physicians use ACE inhibitors to treat heart failure the same way as cardiologists. Although patients of generalists tended to be on higher doses of ACE inhibitors, this may also indicate that generalists were using higher doses to treat higher blood pressure (as indicated by their patients' higher blood pressures) and that cardiologists were using lower doses to treat $\mathrm{CHF}$ in patients who were unable to tolerate adequate dosing.

In contrast to the results of this study, the authors' first study demonstrated differences in knowledge about the indications for ACE inhibitors among specialists. ${ }^{9}$ This national survey of cardiologists, internists, and family practitioners used four clinical vignettes of patients with left ventricular dysfunction to assess the use of ACE inhibitors. The four vignettes reflected the categories of patients that have been shown in randomized clinical trials to benefit from ACE inhibitors. Cardiologists were statistically more likely to choose ACE inhibitors in these vignettes than other specialists (albeit with similar preferences for patients with chronic heart failure). Interestingly, cardiologists rated original research and review articles as more important in making decisions than the other specialists. They were also more likely to titrate the ACE inhibitor to a specific dose while the generalists aimed for relief of symptoms and signs of $\mathrm{CHF}$.

The conclusions drawn from this second study are limited by the low response rate (58\%), which could both introduce bias and limit the generalizability of the findings. The direction of any possible biases are difficult to predict. Even with similar response rates between specialty groups, there may have been differences in the way cardiologists and other physicians responded. The overall low response rate also limits generalizability. For example, because nonresponders were less likely to be board certified, responders may represent a group with more knowledge of recent studies, thus overestimating use in this study. 
The two studies address similar questions, but arrive at different conclusions. ${ }^{7,9}$ Although the survey study suggests that cardiologists use more ACE inhibitors and at more adequate doses, it is limited by nonresponse and by the artificiality of this type of survey research. The second study, based on "real world" treatment, demonstrates no differences but is limited by a lack of information about the indication for the ACE inhibitor, possibly the inability to detect clinically meaningful differences, and the limited generalizability of a single center study. Overall, the studies suggest that cardiologists may be more likely to be using ACE inhibitors as empiric therapy for $\mathrm{CHF}$ and titrating the dose appropriately, and generalist physicians may be more likely to be using ACE inhibitors to treat symptoms and high blood pressure.

Where do we go from here? The results suggest that the use of ACE inhibitor is increasing, at least at one institution. ${ }^{7}$ However, underdosing remains a problem. A recent review suggests that physicians underdose because they believe that high and low doses are equivalent, they base the dose on symptoms, and they limit the dose below those used in randomized trials to avoid side effects. ${ }^{8} \mathrm{~A}$ study is currently underway to assess the issue of proper dosing of ACE inhibitors, ATLAS (Assessment of Treatment with Lisinopril and Survival). Until more is known, physicians should aim to prescribe these agents in doses that were studied and proven effective in randomized clinical trials.

It is still difficult to determine whether or not there is a difference among specialties regarding the use of ACE inhibitors. It is certainly possible that cardiologists are using these agents more appropriately in patients with $\mathrm{CHF}$. Should cardiologists be the sole providers for patients with CHF? The results of the survey showed that only $0.5 \%$ of family practitioners and $4 \%$ of internists cared for as many cardiac patients as cardiologists. ${ }^{9}$ If the generalist sees substantially fewer cardiac patients, is it fair to expect them to be up to date on all aspects of cardiac care? In the age of managed care these questions may already be answered, whether optimal or not. As a result, it is imperative that conclusive information be disseminated to all physi- cians. Based on the information in these studies, this dissemination may best be done from colleague to colleague or through continuing medical education programs. If further research suggests that this dissemination is not effective, then referral of patients with $\mathrm{CHF}$ to a cardiologist would be clinically beneficial for these patients.-MARC A. SCHEINER, MD, Cardiovascular Division, Department of Medicine, and STEPHEN E. KIMMEL, MD, MS, Cardiovascular Division, Department of Medicine, and Center for Clinical Epidemiology and Biostatistics, University of Pennsylvania School of Medicine, Philadelphia.

\section{REFERENCES}

1. American College of Cardiology/American Heart Association Task Force on Practice Guidelines. Guidelines for the evaluation and management of heart failure. J Am Coll Cardiol. 1995;26:1376-98.

2. The SOLVD Investigators. Effect of enalapril on survival in patients with reduced left ventricular ejection fraction and congestive heart failure. N Engl J Med. 1991;325:293-302.

3. The SOLVD Investigators. Effect of enalapril on mortality and the development of heart failure in asymptomatic patients with reduced left ventricular ejection fraction. N Engl J Med. 1992;327:686-91.

4. Pfeffer MA, Braunwald E, Moye LA, et al. Effect of captopril on mortality and morbidity in patients with left ventricular dysfunction after myocardial infarction. Results of the survival and ventricular enlargement trial. The SAVE Investigators. N Engl J Med. 1992; 327:669-77.

5. Young JB, Weiner DH, Yusuf S, et al. Patterns of medication use in patients with heart failure: a report from the registry of studies of left ventricular dysfunction (SOLVD). South Med J. 1995;88:514-23.

6. Bourassa MG, Gurne O, Bangdiwala SI, et al. Natural history and patterns of current practice in heart failure. J Am Coll Cardiol. 1993;22:14-9A.

7. Chin MH, Wang JC, Zhang JX, Lang RM. Utilization and dosing of angiotensin converting enzyme inhibitors for heart failure: effect of physician specialty and patient characteristics. J Gen Intern Med. 1997;12:563-6.

8. Packer M. Do angiotensin converting enzyme inhibitors prolong life in patients with heart failure treated in clinical practice? J Am Coll Cardiol. 1996;28:1323-7.

9. Chin MH, Friedmann PD, Cassel CK, Lang RM. Differences in generalist and specialist knowledge and use of angiotensin converting enzyme inhibitors for congestive heart failure. J Gen Intern Med. 1997; 12:523-30. 